\title{
L'actine cytosquelettique et ses protéines associées II. Aspects pathologiques
}

\section{Abdellatif Fattoum}

\section{ADRESSE}

A. Fattoum : Centre de recherches de biochimie macromoléculaire, CNRS-UPR 1086 , 1919 , route de Mende, 34293 Montpellier

L'actine intervient dans une large variété de processus biologiques. Dans les cellules non musculaires, l'évolution de la bactérie Listeria monocytogenes fournit un exemple de motilité reposant sur l'actine. Les envahisseurs bactériens exploitent activement l'actine de la cellule hôte au cours des phases d'infection et de propagation dans les cellules avoisinantes. In vivo, la régulation de l'association/ dissociation continue des molécules d'actine est strictement contrôlée par un grand nombre de protéines dites associées, agissant individuellement ou de concert pour séquestrer les monomères (thymosine $\beta$ ), coiffer (Cap Z), ponter (filamine), réticuler (actinine $\alpha$ ), fragmenter (gelsoline), ou stabiliser (tropomyosine) les filaments d'actine. Toute perturbation dans l'expression, la structure ou la fonction de ces protéines induit des changements dans le comportement cellulaire, conduisant à la déstructuration architecturale du réseau d'actine.

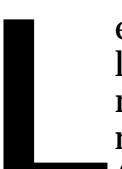

e dynamisme du cytosquelette d'actine est essentiellement fondé sur la vitesse de renouvellement des filaments 100 à 200 fois plus rapide in vivo qu'in vitro), autrement dit sur la modulation de son état intracellulaire, l'actine étant à la fois une molécule cible et médiatrice de la transduction du signal. Les fibroblastes de mammifères en culture, les neutrophiles, les lymphocytes, les extraits cytoplasmiques de plaquettes sanguines et d'œufs de xénopes, les neurones en croissance, les kératinocytes, les hépatocytes, mais aussi les protistes offrent aujourd'hui des modèles de choix pour l'analyse des mécanismes qui contrôlent l'organisation du cytosquelette d'actine et du mouvement cellulaire. Plusieurs maladies, parfois dramatiques, ont pour origine des altérations dans les propriétés structurales et fonctionnelles de différentes molécules cytosquelettiques. Chez l'homme, l'instabilité conformationnelle de certaines protéines spécifiques et leur tendance naturelle à s'autoassocier ou à s'agréger, participant au développement de plusieurs pathologies telles que la maladie d'Alzheimer, la trisomie 21 , les cataractes, la protéinose des alvéoles pulmonaires, I'amyloïdose familiale et autres encéphalopathies transmissibles, telles que I'ESB 
(encéphalopathie spongiforme bovine). Dans cette deuxième partie, nous aborderons les circonstances dans lesquelles des changements qualitatifs ou quantitatifs de l'actine ellemême ou des protéines qui lui sont associées (T ableau I), sont à l'origine de divers aspects physiopathologiques.

\section{Pathologie infectieuse}

L'actine est un acteur extrêmement actif dans la pathologie infectieuse de la plupart des parasites intracellulaires, et plus particulièrement de la bactérie Listeria monocytogenes, responsable de la listériose humaine. Cette infection touche préférentiellement les enfants en bas âge et les personnes âgées, et provoque des avortements spontanés chez les femmes enceintes ainsi que des méningoencéphalites chez les patients immunodéprimés. Durant le pic de l'épidémie ayant récemment sévi en France (février/ mars 2000), une cinquantaine de personnes ont été infectées et 10 décès ont été rapportés. La dernière épidémie remonte à 1992, et fit 280 victimes contaminées et 63 morts. Les risques avérés de cet agent pathogène relèvent de la sécurité sanitaire des aliments et de la santé publique. Une meilleure connaissance de son mode d'action et des facteurs protéiques impliqués dans sa force de propulsion dans les cellules hôtes permettrait une meilleure compréhension de la motilité cellulaire en général, et de la migration des cellules métastatiques en particulier. Les rôles de l'actine, ainsi que celui des protéines comme la zyxine et l'ezrine dans certains aspects de la motilité ont été récemment décrits par RM Golsteyn et al. [1], et nous ne les développerons pas dans cet article.

Au cours de la phase d'invasion, les bactéries font appel à des composants protéiques qui leur permettent de détourner à leur profit l'actine cytosquelettique de la cellule infectée. L'actine leur sert alors de force de mouvement, facilitant leur adhérence cellulaire, leur internalisation, leur réplication, leur survie dans la cellule hôte et enfin leur propagation vers les cellules avoisinantes. L'avantage d'un tel mode d'expansion est que la bactérie s'affranchit totalement de la rencontre avec le système immunitaire de l'espace intracellulaire, en passant d'une cellule à l'autre quasiment sans jamais quitter le cytoplasme. La motilité intracellulaire de ces parasites nécessite des interactions protéine-protéine, par l'intermédiaire de séquences riches en proline. Chez L. monocytogenes, I'ActA, protéine spécifiquement exprimée à la surface de la bactérie, se fixe au segment aminoterminal de la VASP (vasodilator-stimulated-phosphoprotein), qui s'associe à son tour à un nombre restreint de protéines pour déclencher la polymérisation de l'actine cytosquelettique du côté postérieur de la bactérie. Cette réaction est suivie de l'assemblage unidirectionnel et de la formation, dans le sillage de la bactérie, d'une queue plus ou moins longue, en forme de comète, polarisée (les extrémités barbues des filaments étant orientées vers la surface de la bactérie), et très dense. Cette queue est composée de filaments d'actine interconnectés par des protéines de pontage, telles que l'alpha-actinine et la plastine (figure 1). Les protéines impliquées dans l'attachement des filaments à la bactérie sont parfois différentes. Chez Shigella flexneri, l'agent de la dysenterie, la protéine de surface ICSA ne possède aucune homologie de séquence avec ActA. Elle induit l'assemblage de l'actine et la motilité de la bactérie en se liant spécifiquement à la protéine $\mathrm{N}$ WASp, membre de la famille du même nom et activée par $\mathrm{Cdc42}$. Chez l'homme, les mutations dans le gène de N-WASp sont responsables du syndrome de Wiskott-Aldrich, une maladie rare liée à I' $X$, qui se caractérise par un déficit immunitaire sévère et un défaut dans le développement des plaquettes [1]. Dans tous les cas, la polymérisation de l'actine est essentielle pour provoquer la poussée vers l'avant de la bactérie, sa vitesse de déplacement étant proportionnelle à la longueur de la queue formée. Cette organisation particulière autour de l'actine est renforcée par un complexe multiprotéique de sept sous-unités, renfermant un duo d'actines non conventionnelles dénommé Arp2/ 3 (actin-related proteins), capable de stimuler la nucléation de l'actine-G, de permettre sa liaison avec domaine amino-terminal de l'ActA (chez Listeria), de reconsti- tuer et de contrôler, avec l'apport nécessaire des phospholipides, l'assemblage des filaments d'actine synthétisés de novo [2]. D'autres molécules du cytosquelette participent à l'efficacité de l'invasion bactérienne: une protéine de coiffe (capping protein) et la cofiline/ ADF (actin-depolymerising-factor), qui se fixe à l'actine et assure le recyclage de ses filaments en les dépolymérisant. La cofiline/ ADF, en synergie avec la profiline qui lie également I'actine-G, joue un rôle clé dans l'augmentation du renouvellement des filaments d'actine et donc de la vitesse du déplacement de la bactérie [3].

Chez la plupart des bactéries, les phases d'adhérence et de pénétration sont précédées d'une sécrétion de facteurs de virulence, d'une activation des voies de transmission du signal par les ions calcium, les phospholipides, la phosphorylation des tyrosines et les petites GTPases (Rac, Rho et (dc42), et d'une perturbation d'un grand nombre de protéines associées à l'actine, situées au niveau de la membrane plasmique [4].

Cette stratégie d'infection cellulaire est également utilisée en partie par deux grandes familles d'agents pathogènes: d'une part les virus de la variole, de la vaccine, du SIDA et de la rage, et d'autre part les parasites protozoaires tels que Plasmodium falciparum et Theileria parva, responsables respectivement du paludisme chez l'homme et de la leucémie chez les bovins. La détection de l'actine dans les virions purifiés du rétrovirus $\mathrm{VIH}$ 1 laisse supposer que le cytosquelette est impliqué dans la transmission intercellulaire du virus. En effet, le traitement par la cytochalasine D des cellules lymphoïdes et des monocytes infectés par le VIH-1 déstabilise les structures microfilamentaires, et conduit à une diminution partielle de la production du virus. De plus, la délétion du domaine nucléocapsidique de la protéine Gag du virus VIH -1 réduit considérablement la distribution, I'assemblage et la production des particules virales dans les celIules T $\mathrm{CD}^{+}{ }^{+}[5]$. En raison des similitudes entre la motilité des bactéries et celle des virus, on peut conclure que ces différents agents pathogènes développent un mécanisme commun, fondé sur le recrutement et I'exploitation du cytosque- 
Tableau I. Principales classes de protéines associées à l'actine.

\begin{tabular}{|c|c|}
\hline Protéine & Mode d'action \\
\hline & Sur le monomère \\
\hline \multirow{4}{*}{$\begin{array}{l}\text { Classe I : } \\
\text { Thymosine } \beta 4 \\
\text { Profiline } \\
\text { DNase I } \\
\text { Cc globuline }\end{array}$} & \\
\hline & $\rightarrow$ séquestration \\
\hline & \\
\hline & Sur le filament \\
\hline \multicolumn{2}{|l|}{ Classe II : } \\
\hline Gelsoline & $\rightarrow$ coiffe, nucléation \\
\hline Villine & et/ou fragmentation \\
\hline $\begin{array}{l}\text { Scinderine } \\
\text { Fraqmine/Severine }\end{array}$ & \\
\hline Adseverine/Scinderine & \\
\hline CapZ ( $\beta$ actinine) & \\
\hline $\begin{array}{l}\text { gCap 39/MCP } \\
\text { Tropomoduline }\end{array}$ & \\
\hline (ADF)/Cofiline & \\
\hline Actophorine & \\
\hline Dépactine & \\
\hline Tensine & \\
\hline \multicolumn{2}{|l|}{ Classe III : } \\
\hline ABP-280/Filamine & $\rightarrow$ réticulation, rigidifica- \\
\hline$\alpha$-actinine & tion et formation de \\
\hline $\begin{array}{l}\text { ABP-120 } \\
\text { Spectrine/Fodrine }\end{array}$ & $\begin{array}{l}\text { Talsceaux paralleles } \\
\text { (structures anisotropes) }\end{array}$ \\
\hline Plastine/Fimbrine & ou de réseaux tridimen- \\
\hline Fascine & sionnels (structures iso- \\
\hline Actinogéline & \\
\hline $\begin{array}{l}\text { Arp } 2 / 3 \\
\text { VASP }\end{array}$ & \\
\hline \multicolumn{2}{|l|}{ Classe IV : } \\
\hline$\alpha$-actinine & $\rightarrow$ ancrage \\
\hline Filamine & membranaire \\
\hline Vinculine & (terminal ou latéral) \\
\hline $\begin{array}{l}\text { Dystrophine } \\
\text { Actolinkine }\end{array}$ & \\
\hline Ponticuline & \\
\hline Catinine & \\
\hline Taline & \\
\hline Ankyrine & \\
\hline $\begin{array}{l}\text { Spectrine/Fodrine } \\
\text { Protéine } 4.1\end{array}$ & \\
\hline Tensine & \\
\hline $\begin{array}{l}\text { Adducine } \\
\text { ERM }\end{array}$ & \\
\hline \multicolumn{2}{|l|}{ Classe V: } \\
\hline Tropomyosine & $\rightarrow$ stabilisation \\
\hline ABP - 280/Filamine & \\
\hline Caldesmone & \\
\hline \multicolumn{2}{|l|}{ Classe VI : } \\
\hline Myosine & $\rightarrow$ transduction \\
\hline Tropomyosine & mécanochimique, \\
\hline Troponine I,T,C & contraction \\
\hline Caldesmone & \\
\hline Calponine & \\
\hline
\end{tabular}

Toutes ces protéines sont ubiquitaires et relativement conservées. Elles renferment au minimum un site de liaison à l'actine et contribuent à la dynamique du filament au cours de la motilité cellulaire. Plusieurs évènements qui s'effectuent à la surface de la membrane (expression de molécules, coiffe, extension, rétraction, infection virale) dépendent étroitement de l'intégrité du cytosquelette d'actine. La plupart des protéines liant l'actine participent à la transduction du signal à travers les GTPases, coiffent l'extrémité barbue du filament et possèdent une activité biologique modulée par le calcium, le $\mathrm{pH}$, les polyphospho-inositides et la phosphorylation. Certaines de ces protéines sont structurellement reliées et remplissent parfois des fonctions similaires vitales, probablement pour éviter une instabilité génétique susceptibles de provoquer des mutations catastrophiques pour la cellule. L'altération de l'expression ou de la fonction in vivo de ces molécules peut affecter profondément l'état de l'actine intracellulaire. Les anomalies génétiques de certaines protéines associées à l'actine engendrent des désordres physiologiques débilitants et parfois fatals. C'est le cas de la dystrophine dans la myopathie de Duchenne, de la synapsine dans le syndrome de Rett et de la spectrine dans les anémies hémolytiques héréditaires. Plusieurs de ces protéines sont directement ou indirectement impliquées dans d'autres maladies humaines et peuvent être utilisées comme des marqueurs spécifiques de la différenciation et représenter des outils précieux dans le diagnostic précoce de la cancérisation.

lette d'actine, en vue de faciliter leur essaimage direct parmi les cellules qu'ils envahissent.

\section{Pathologie tumorale}

Dans les pathologies tumorales, l'actine semble jouer un rôle clé au cours du processus invasif. En effet, la transformation cellulaire maligne se caractérise par des altérations dans la morphologie cellulaire, I'adhérence au substratum et la motilité. Elle s'accompagne le plus souvent d'une désorganisation des réseaux et des faisceaux d'actine, et d'une diminution de la quantité de plusieurs de ses protéines associées, considérées comme des facteurs potentiels de régulation de la croissance cellulaire et de la différenciation.

Le mouvement cellulaire est fondé sur des réseaux à configurations multiples, constitués de filaments d'actine, et représentant une véritable charpente pour le cytoplasme périphérique. Ces réseaux sont constamment en interaction avec des protéines associées à l'actine, leur rôle étant de faire et de défaire ces structures pour assurer le cycle d'assemblage/ désassemblage du filament, nécessaire au déplacement de la cellule. En général, la transforma- 


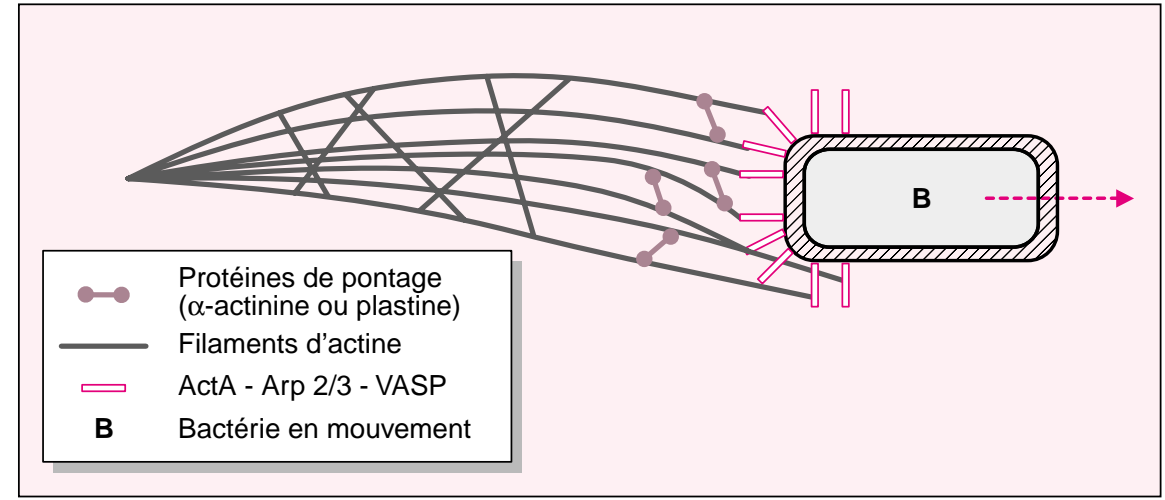

Figure 1. Représentation schématique de l'organisation des filaments d'actine au cours du mouvement de Listeria monocytogenes. Arp 2/3 induit la nucléation des nouveaux filaments, s'associe et se dissocie de la protéine bactérienne ActA, elle-même liée à la protéine VASP. Cette dernière est connectée à la profiline qui, avec la cofiline, stimule la polymérisation de l'actine. L' $\alpha$-actinine ponte les filaments d'actine et les transforme en réseaux rigides facilitant la production de force et la poussée de la bactérie vers l'avant. L'allongement des filaments est interrompu par les protéines coiffantes qui bloquent les extrémités barbues.

tion maligne d'un tissu altère l'expression et la distribution des protéines structurales associées à I'actine et affecte l'architecture du cytosquelette. Des observations parfois contradictoires ont été rapportées entre l'expression de ces protéines ou leur isoformes (leur contrôle peut être positif ou négatif), et le potentiel de transformation des cellules tumorales. Ces résultats soulignent la complexité du processus de maintien de l'équilibre instable qui s'instaure entre les différents composants protéiques du cytosquelette et la migration cellulaire. Dans des lignées sarcomateuses de rats, les cellules ayant des filaments fins et courts d'actine ont généralement le potentiel métastatique le plus élevé. Dans les cellules de lymphomes T BW5147, I'analyse de la motilité a montré qu'une polymérisation accrue d'actine est indispensable à la formation de pseudopodes, à l'infiltration cellulaire dans les tissus, et éventuellement au développement des métastases. Par ailleurs, dans les mélanomes B16 de souris, la présence du variant d'actine $\beta \mathrm{m}$ coexprimé au côté des deux isoformes $\beta$ et $\gamma$, est inversement liée à la désorganisation des fibres de tension et au potentiel de transformation de ces cellules. Ce variant est davantage exprimé dans les cellules ayant une faible capacité métastatique. L'expression de $\mathrm{m} / \mathrm{sn}^{\circ} 2$, vol. 17, février 2001
Les changements dans les propriétés structurales et fonctionnelles de l'actine ne sont pas seuls à pouvoir signaler l'apparition d'un phénomène malin. Plusieurs protéines associées à l'actine peuvent en effet produire ou traduire la malignité d'une cellule. C'est le cas de la thymosine $\beta-4$, une petite protéine ubiquitaire de 43 acides aminés, capable de séquestrer jusqu'à $50 \%$ de l'actine G-ATP, et de contrôler son état de polymérisation/ dépolymérisation dans des cellules mobiles telles que les polymorphonucléaires et les plaquettes sanguines. L'augmentation du taux d'expression de thymosine $\beta-4$ dans des lignées cellulaires métastatiques (mélanomes B16 de souris et A375 humaines) et dans les tissus de carcinomes pulmonaires ou colorectaux par rapport à des tumeurs malignes sans métastases, résulterait d'un dysfonctionnement dans l'organisation des microfilaments, produisant des signaux susceptibles d'induire des changements dans I'adhérence, la croissance et la capacité métastatique des cellules [8]. Par ailleurs, le taux de la thymosine $\beta-15$, un nouveau membre de la famille thymosine- $\beta$, serait élevé dans les cancers métastatiques de la prostate [9]. Cette protéine représente, comme d'autres types de protéines associées à l'actine, un marqueur biochimique potentiel de la progression du cancer de la prostate, qui constitue la deuxième cause de mortalité chez les hommes âgés.

Dans les cellules 3T3 BALB/ c ayant subi une transformation par le virus simien SV40, le taux d' $\alpha$-actinine exprimée est six fois inférieur à celui des cellules normales. Lorsque l'expression de la protéine est restaurée par injection d'ADN c d' $\alpha$-actinine-1 (non musculaire), la tumorigénicité de ces cellules est fortement réprimée. Ainsi, I' $\alpha$-actinine qui représente un composant structural majeur des microfilaments et une protéine de pontage, d'ancrage et de stabilisation des filaments d'actine, pourrait à elle seule avoir une capacité de répression effective sur le phénotype transformé. L'actinine-4, une forme récemment décrite d' $\alpha$ actinine, est impliquée dans la motilité cellulaire et l'invasion cancéreuse. Elle se lie aux filaments d'actine dans des compartiments sub- 
cellulaires différents de ceux de l'actinine-1. L'actinine-4 est concentrée plus particulièrement dans le cytoplasme, et sa translocation vers le noyau, suite à l'inhibition de la 3phosphatidylinositol kinase ou à la dépolymérisation de l'actine par la cytochalasine $D$, est étroitement associée au mécanisme d'invasion cellulaire qui se traduit par un très mauvais pronostic dans les cancers du sein et de la vessie. L'actinine-4 semble donc régler le cytosquelette d'actine et accroître la motilité des cellules cancéreuses. Son inactivation par transfert dans le noyau abolit le potentiel métastatique des cancers humains [10]. Elle représente aussi un nouveau marqueur biologique précoce des métastases, prédictif de la rechute de cancer du sein.

Dans $60 \%$ à $90 \%$ des tumeurs au cours de la carcinogenèse du sein, du colon, de l'estomac, de la vessie, de la prostate et du poumon, la perte de l'expression de la gelsoline peut être totale [11]. La villine, dont l'expression est spécifique du tissu, est particulièrement abondante dans les microvillosités des bordures en brosse de l'épithélium intestinal et des reins. Elle peut être modifiée dans sa composition et dans sa distribution pendant la transformation cellulaire et constitue de ce fait un marqueur de choix des états prénéoplasiques et néoplasiques du côlon. En vertu de son activité de sevrage (fragmentation) vis-à-vis de l'actine, la villine peut intervenir bénéfiquement dans le remodelage du cytosquelette au cours de situations physiologiques particulières telle que la réparation des lésions de l'épithélium intestinal [12].

La caldesmone et la calponine sont deux protéines fortement associées à l'actine, qui occupent un rôle déterminant dans la régulation de la contraction du muscle lisse. Dans une revue précédente [13], nous avons décrit quelques aspects de la pathologie de ce tissu en relation avec ces deux protéines dont la présence est considérée comme critique, tant pour la stabilité et l'intégrité structurale du cytosquelette d'actine que pour le contrôle de la contractilité cellulaire et la motilité. Plus récemment, il a été rapporté que la baisse du taux d'expression de la cal- cholélithiase est en rapport avec le dysfonctionnement du sphincter d'Oddi. Celui-ci voit sa vitesse de contraction augmenter, favorisant ainsi la stase biliaire et conduisant à la nucléation et à la rétention des microcristaux qui précipitent et s'agglomèrent pour former des calculs [14]. Par ailleurs, la calponine h1 basique qui s'exprime normalement dans le myomètre et dans le leiomyome de l'utérus humain est peu ou pas représentée dans le leiomyosarcome. La surexpression de la calponine $h 1$ basique dans ce tissu réduit fortement l'activité proliférative et la tumorigénicité des cellules transfectées, très probablement en inhibant les voies de signalisation qui contrôlent I'adhérence de la cellule au substrat, sa forme et son déploiement, processus intimement liés au cytosquelette d'actine. La calponine peut donc fonctionner comme un suppresseur de tumeurs, et ouvre la voie à une nouvelle thérapie potentielle pour ce néoplasme extrêmement malin de l'utérus [15].

\section{Pathologie endocrinienne}

Différents travaux font état de l'effet de l'insuline sur les propriétés structurales de la cellule et plus particulièrement sur le degré de polymérisation de l'actine et sa distribution intracellulaire. La présence d'insuline dans le cytosol réduit très sensiblement la concentration de l'actine-G, suggérant une stimulation de la polymérisation de la molécule par cette hormone et un réarrangement rapide des filaments du cytosquelette sous la membrane plasmique, non loin du récepteur de l'insuline. Les réseaux de filaments d'actine corticale et leur réorganisation sont d'une importance capitale dans la propagation des signaux métaboliques et mitogéniques que l'insuline envoie après sa fixation au récepteur-tyrosine kinase, conduisant à la stimulation du transport du glucose et à l'activation d'une cascade de protéine kinases. La dépolymérisation des filaments d'actine par la cytochalasine D, la latrunculine $A$ ou la toxine clostridiale C2, perturbe la sécrétion de l'insuline, inhibe la translocation du principal transporteur de glu- cose GLUT4 vers la membrane cellulaire et empêche le transport du glucose dans les cellules musculaires $L 6$ différenciées et les adipocytes 3T 3-L 1[ 16].

Enfin, le taux élevé de glucocorticoïdes chez des sujets atteints du syndrome de Cushing ou chez des patients ayant suivi un traitement thérapeutique prolongé contre l'asthme ou l'arthrite rhumatoïde, a depuis longtemps été associé au développement de l'ostéoporose. La dexaméthasone, utilisé à des doses supraphysiologiques, réduit fortement la production de prostaglandine (PGE2) et la synthèse de I'ADN, modifie la morphologie celIulaire, défait l'architecture des fibres de tension de l'actine et retarde la croissance des ostéoblastes, contribuant ainsi à la réduction de la formation et de la qualité de l'os. L'addition de PGE2 exogène dans des cultures d'ostéoblastes traités par la dexaméthasone induit le recouvrement de l'architecture de l'actine et du phénotype cellulaire [17].

\section{Pathologie sensorielle et neurodégénérative}

Parmi les partenaires majeurs de l'actine, la myosine représente le moteur moléculaire le plus abondant et le mieux connu [18]. Elle est impliquée dans la presque totalité des mouvements cellulaires fondés sur les filaments d'actine. On dénombre actuellement 15 classes ou sous-familles de myosine, structuralement distinctes, dites non conventionnelles par opposition à la myosine II conventionnelle. Des analyses génétiques ont montré la présence de mutations ponctuelles dans les gènes codant pour la myosine de classe II, et d'autres protéines sarcomériques. Ces mutations sont situées au niveau du domaine catalytique, et altèrent directement le mécanisme d'interaction entre l'actine et la myosine, entraînant une désorganisation myofibrillaire, une surproduction mécanique compensatoire, une augmentation de la masse ventriculaire et des dysfonctionnements systolique et diastolique (voir plus loin). Les myosines non conventionnelles se distinguent par leur rôle essentiel dans la transduction de messages sensoriels 
[19]. En effet, des mutations dans le gène codant pour la myosine VII sont à l'origine de déficiences auditives et visuelles majeures, chez I'homme et chez la souris, comme l'illustre notamment la forme la plus fréquente du syndrome de Usher de type $1 \mathrm{~B}$, responsable de la plupart des cas de surdité associée à une cécité. Ce double déficit neurosensoriel est dû à une atteinte de l'organe de Corti (organe récepteur de l'audition) et à une rétinite pigmentaire. L'isomyosine VII joue un rôle primordial dans le maintien et l'intégrité des fuseaux microfilamentaires d'actine dans les cellules ciliées de la structure interne de l'oreille, et est aussi impliquée dans le phénotype des souris shaker-1, atteintes de surdité. L'altération du gène de la myosine $V$, initialement mise en cause dans le phénotype de pigmentation anormale chez les souris dilute, a été récemment impliquée dans la maladie de Griscelli, caractérisée par un déficit immunitaire cellulaire, un dysfonctionnement neurologique et un albinisme partiel, dû à un défaut de transport des granules pigmentés dans les mélanocytes. La myosine III a été localisée dans les cellules photoréceptrices de la drosophile, et des mutations dans le gène correspondant $(\mathrm{NinaC})$ induisent une dégénérescence de la rétine. Enfin, chez la souris Snells Waltzer, une mutation récessive dans le gène de la myosine VI conduit à la présence d'une forme tronquée de la molécule dans l'oreille interne et provoque une surdité.

II existe également des liens spécifiques entre des protéines associées à l'actine et la neurofibromatose de type 2 , une maladie humaine héréditaire touchant le système nerveux, et caractérisée par la présence de neurinomes et de multiples meningiomes. Le gène responsable de cette maladie nerveuse code pour la merline, une protéine récemment identifiée, et structuralement similaire aux diverses protéines ERM (ezrine, radixine, moesine) qui renferment des sites susceptibles de lier à la fois la membrane plasmique et le cytosquelette [20]. Lors de la fragmentation cellulaire, le domaine de la merline, homologue à celui des protéines ERM, cosédimente avec l'actine F. II est capable, à lui seul, de supprimer le phénotype tumoral des cellules NIH/ 3T 3 transformées par l'oncogène ras.

L'une des fonctions importantes de la gelsoline sécrétée réside dans son interaction avec l'acide lysophosphatidique, présent dans divers sites extracellulaires. Ainsi, cette protéine modulatrice de l'actine pourrait exercer un rôle de transporteur de lipides. Elle est potentiellement impliquée dans un ensemble de maladies dégénératives, regroupées sous le terme d'amyloïdose, dans lesquelles se forment des dépôts de protéines fibrillaires, qui dérivent de protéines biochimiquement distinctes. L'amyloïdose systémique de la gelsoline, appelée aussi amyloïdose familiale de type finnois (FAF), est une maladie héréditaire de type autosomique dominant, dont les symptômes sont une polyneuropathie associée à une dystrophie cornéenne, à des modifications cutanées (peau hyperélastique) et à des complications rénales. Cette maladie, qui touche préférentiellement les neurones, semble être le résultat d'une combinaison entre la formation des dépôts amyloïdes et la perte d'activité de la gelsoline. L'immunodétection du fragment amyloïde $65 \mathrm{kDa}$, spécifique de la maladie, dans le plasma et le liquide cérébrospinal, ainsi que d'autres fragments de la gelsoline mutée retrouvés dans les urines, s'est révélée être un moyen utile pour diagnostiquer la maladie liée à la FAF [21]. Toutes ces observations révèlent l'importance physiopathologique de la gelsoline et son rôle spécifique dans la régulation de la différenciation neurale et le développement normal du cerveau.

\section{Pathologie vasculaire}

Lorsque les cellules du muscle cardiaque sont soumises à une anoxie ou à une ischémie, elles relâchent des composants intracytoplasmiques, suite à des lésions de la membrane cellulaire. Selon un nouveau test clinique reposant sur l'actine, il est possible d'établir une nouvelle classification des maladies coronariennes qui varient de l'infarctus à l'angine de poitrine itérative, avec infarcissement léger du tissu myocardique. Les deux tiers des patients souffrant d'angine de poitrine prolongée présentent une augmentation de l'actine- $\alpha$ circulante [22]. Le gène codant pour l'actine- $\alpha$ cardiaque a été incriminé dans les formes autosomiques dominantes de certaines maladies cardiaques humaines. Ces cardiomyopathies sont caractérisées par une dilatation des cavités ventriculaires et une baisse de la contractilité du myocarde, pouvant conduire à l'insuffisance cardiaque réfractaire progressive et parfois à la mort subite, représentant ainsi une indication majeure de transplantation cardiaque [23]. Les deux mutations faux sens ( $\mathrm{Arg} 312 \mathrm{H}$ is et Glu361Gly), situées au niveau des exons 5 et 6 , affectent les sites d'attache de l'actine aux lignes $Z$ et aux disques intercalaires. Plus récemment, une nouvelle mutation (Ala295Ser) située dans l'exon 5 (non loin des deux mutations précédentes et à proximité du site putatif de fixation de la myosine) a été décrite dans une cardiomyopathie hypertrophique [24]. II s'agit par conséquent du premier gène sarcomérique décrit, dans lequel les mutations arborées sont responsables de deux cardiomyopathies différentes, I'une dilatée et I'autre hypertrophique. Par ailleurs, plus de trente mutations différentes ont été répertoriées au niveau du gène de la chaîne lourde ß de la myosine chez des patients atteints de cardiomyopathies hypertrophiques familiales, la plus sévère étant la mutation Arg403GIn, située dans une boucle du site d'interaction de l'actine avec la myosine, au niveau du domaine moteur S-1. Ces mutations perturbent la translocation des filaments d'actine, en provoquant I'inhibition de l'activité actomyosine ATPase, et la réduction substantielle de la vitesse de motilité des filaments de myosine purifiés in vitro [25]. D'autres mutations, affectant sévèrement la fonction cardiaque, ont été mises en évidence dans des protéines sarcomériques appartenant soit au filament fin, soit au filament épais: troponines T et I, $\alpha$-tropomyosine, chaînes légères de la myosine, protéine-C [26]. La mutation de la titine, protéine géante découverte récemment, semble augmenter son affinité de fixation à l' $\alpha$-actinine, modulant ou altérant la transmission de force à travers le disque Z [27]. Des analyses immunochimiques effectuées à partir 
du sérum de malades cardiaques, ont mis en évidence un taux de troponines $T$ et I supérieurs à ceux de la créatine kinase et de la lactate déshydrogénase. Ces résultats indiquent que la troponine peut servir de marqueur dans le diagnostic de cette forme de cardiomyopathie idiopathique [28].

\section{Conclusions et perspectives}

Chez les organismes eucaryotes, l'actine est l'une des protéines les plus abondantes et les mieux conservées au cours de l'évolution. Sa principale propriété réside dans sa capacité de s'autoassocier de manière réversible. In vivo, l'organisation spatio-temporelle de l'actine est contrôlée par des signaux provenant essentiellement de la surface cellulaire. Le moindre dérèglement dans les voies de signalisation, suivie de l'activation ou de la désactivation des protéines clés du cytosquelette, induit une désorganisation du réseau microfilamentaire, qui peut se traduire par un changement général dans la fonction physiologique de l'organisme et conduire à des désordres incompatibles avec la survie de l'individu. Etant donné le rôle majeur des microfilaments dans la régulation de l'activité métabolique, le changement de forme, la croissance ou la mort programmée de la cellule, la molécule d'actine occupe une place de choix dans les investigations concernant la sénescence des tissus, et leur incidence éventuelle sur la fréquence des cancers chez les sujets âgés [29]. Enfin, on sait depuis peu que le cytosquelette d'actine joue un rôle essentiel dans la formation des «synapses immunologiques» et la signalisation par la voie du récepteur $T$ de l'antigène, au cours de l'activation lymphocytaire et de la réponse immune [30]. Ces résultats ouvrent la voie à des études concernant les communications transmembranaires et intercellulaires, qui pourraient conduire au développement de thérapies immunologiques. Le cytosquelette est donc un site privilégié de communications et de renseignements, orchestrés par des signaux sophistiqués, et représente un carrefour décisionnel incontournable, où
Iule. Cependant, si I'on s'accorde à dire que des progrès substantiels ont été réalisés depuis une vingtaine d'années concernant l'actine et ses protéines associées, plusieurs questions fondamentales subsistent. Pourquoi une cellule a-t-elle besoin de plus d'une protéine de réticulation, de séquestration, de fragmentation ou de transduction (T ableau I) ? Pourquoi une cellule a-t-elle besoin d'autant d'isoformes d'actine (six pour les vertébrés à sang chaud) et de moteurs protéiques (environ seize isomyosines) ? Pourquoi le système microfilamentaire est-il redondant avec des protéines qui, en dehors de leurs propriétés spécifiques, partagent des fonctions similaires ? Comment s'effectue la coordination rapide et précise des différentes actions des composants du puzzle cellulaire ? Comment la cellule utilise-telle les différents mécanismes de régulation pour moduler localement la dynamique de l'actine et influencer le comportement cellulaire? Quel est l'impact du repliement et de la distribution tridimensionnelle de certaines protéines majeures du cytosquelette, y compris l'actine, sur l'origine et l'évolution de certaines maladies génétiques humaines ? Enfin, peut-on comparer les observations réalisées in vitro avec des situations in vivo?

Nous sommes encore loin de connaître tous les aspects des protéines associées à l'actine, et l'on suspecte que de nouveaux membres de cette famille seront identifiés dans un avenir proche. Le regard que nous portons sur ces protéines est en pleine évolution, et les recherches concernant le contrôle des complexes multiprotéiques du cytosquelette in vivo, connaîtra sans doute un développement spectaculaire à l'aube du troisième millénaire. Ces avancées sont possibles grâce à la maîtrise de technologies convergentes classiques de bio-physicochimie et d'approches géniques beaucoup plus récentes.

Le second défi à relever dans les dix prochaines années est l'élucidation des mécanismes par lesquels les RhoGTPases orchestrent I'organisation du cytosquelette d'actine, phénomène essentiel pour la régulation de processus biologiques complexes, tels que la détermination de la forme, de
I'adhérence et de la motilité cellulaires. II conviendrait maintenant d'orienter davantage les recherches vers l'étude des interactions moléculaires que vers les observations réalisées jusqu'à présent à l'échelle cellulaire ou tissulaire. Ces approches pourraient permettre de détecter le défaut responsable d'une maladie chez un patient, et de mettre en œuvre des stratégies thérapeutiques novatrices et adaptées à chaque cas. Enfin, il faut encourager l'émergence de réseaux de recherche associant des fondamentalistes et des cliniciens autour de programmes ciblés sur les interactions moléculaires, les anomalies génétiques et la physiopathologie du cytosquelette, dans l'espoir d'aboutir à des thérapies pharmacologiques ciblées. Le séquençage récent de la quasi-totalité du génome humain ne peut qu'encourager l'ensemble de ces recherches. Ainsi, le traitement à la carte de la maladie d'Alzheimer, de Parkinson, de l'hypertension, de l'asthme, du diabète ou du cancer, longtemps considéré comme une utopie, pourra bientôt être envisageable

\section{RÉFÉRENCES}

1. Golsteyn RM, Arpin, M Friederich, E Louvard, D. Les protéines du cytosquelette d'actine: bien placées pour la motilité. M ed Sci $2000 ; 16$ : 722-31.

2. Steffen $P$, Schafer DA, David V, Gouin E, Cooper JA, Cossart P. Listeria monocytogenes ActA protein interacts with phosphatidylinositol 4,5-biphosphate in vitro. Cell Motil cytoskeleton $2000 ; 45$ : 58-66.

3. Loisel TP, Boujemaa R, Pantaloni D,Carlier MF. Reconstitution of actin-based motility of Listeria and Shigella using pure proteins. Nature 1999 ; 401 : 613-6.

4. Tapon N, Hall A. Rho, Rac and $\mathrm{Cdc} 42$ GTPases regulate the organization of the actin cytoskeleton. Curr O pin Cell Biol 1997 ; 9: 86-92

5. Liu B, Dai R, Tion CJ, Dawson L, Gorelick $R, Y u X F$. Interaction of the human Immunodeficiency virus type 1 nucleocapsid with actin. J Virol 1999; 73: 2901-08.

6. Janmey PA, Chaponnier C. Medical aspects of the actin cytoskeleton. Curr 0 pin Cell Biol 1995 ; 7 : 111-7. 


\section{RÉFÉRENCES}

7. Economidou-Karaoglou A, Lans M, Taper HS, Michaux JL, Roberfroid M. Variations of serum alkalin DNase activity: a new means to assess early detection of relapse in patients treated for acute non-lymphoblastic Leukemia. Blood $1989 ; 74$ : 2730-2.

8. Clark EA, Golub TR, Lander ES, Hynes RO. Genomic analysis of metastasis reveals an essential role for RhoC. Nature 2000 ; $406: 532-5$.

9. Bao L, Loda M, Janmey PA, Stewart R, Anand-Apte $B$, Zetter BR. Thymosin beta 15: a novel regulator of tumor cell motility upregulated in metastatic prostate cancer. $N$ at M ed 1996; 2 : 1322-8.

10. Honda K, Yamada T, Endo R, et al. Actinin-4, a novel actin-bundling protein associated with cell motility and cancer invasion. J Cell Biol 1998 ; 140: 1383-93.

11. Kwiatkowski D. Functions of gelsolin: motility, signaling, apoptosis, cancer. Curr op Cell Biol 1999; 11: 103-8.

12. H o SB. Cytoskeleton and other differenciation markers in the colon. J Cell Biochem 1992 ; $16 \mathrm{G}: 119-28$.

13. Fattoum A. Mécanismes moléculaires de régulation de la contraction du muscle lisse. M ed Sci 1997 ; 13: 777-89.

14. Lu W, Tang D, Cao S, Yu CH. The study on the relationship between the expression of calponin and gallstone formation. Journal of T ongji M edical University 1997 ; 17 : 86-9.

15. Horiuchi A, Nikaido T, Taniguchi $S$, Fujii S. Possible role of calponin hl as a tumor suppressor in human uterine leiomyosarcoma. J Nat Cancer Inst 1999; 91 : 790-6.

16. Khayat ZA, Tong $P$, Yaworsky K, Bloch RJ, Klip A. Insulin-induced actin fillament remodeling colocalizes actin with phosphatidylinositol 3-kinase and GluT 4 in L 6 myotubes. J Cell Sci 2000 ; 113: 279-90.

17. Fulford $M$, Appel $R$, Kumegawa $M$, Schmidt J. Effect of dexamethasone on proliferating osteoblasts: inhibition of prostaglandine $E_{2}$ synthesis, DNA synthesis and alterations in actin cytoskeleton. Exp Cell Res 1992 ; 203: 150-6.

18. Eldin $\mathrm{P}$, Cornillon $\mathrm{B}$, Mornet $\mathrm{D}$, Léger Jj. Une nouvelle jeunesse pour les myosines. $M$ ed Sci 1995; 11: 1005-16.
19. Mervall V, Post $P$, Mooscker MS. Unconventional myosins in cell movement, membrane traffic and signal transduction. Science 1998 ; 279: 527-33.

20. Tsukita S, Yonemura S. Cortical actin organization: lessons from ERM (Ezrin/Radixin/ Moesin) proteins. I Biol Chem 1999 ; 274: 34507-10.

21. Wetsberg JA, Zhang KZ, Andersson LC. Regulation of neural differentiation by normal and mutant (G654A, amyloidogenic) gelsolin. FASEB ] 1999 ; 13: 1621-6.

22. Aranéga $A E$, Reina $A$, Velez $C$, Alvarez $L$, Melguizo $C$. Circulating $\alpha$-actin in angina pectorís. J M ol Cell Cardiol 1993 ; 25 ; 15-22.

23. Tesson F, Charron P, Schwartz K, Komajda M. Génétique des cardiomyopathies dilatées. M ed Sci 1999; 15: 369-75.

24. Mogenson J, Klaussen IC, Pedersen AK, Egeblerd $H$, et al. $\alpha$-cardiac actin is a novel disease gene in familial hypertrophic cardiomyopathy. J Clin Invest 1999 ; 103: R39R43.

25. Bartegi A, Roustan C, Chavanieu A, Kassab R, Fattoum A. Interaction of F-actin with synthetic peptides spanning the loop region of human cardiac $\beta$-myosin heavy chain containing Arg403. Eur J Biochem $1997 ; 250$ : 484-91.

26. Bonne G, Carrier L, Richard $P$, et al. Génétique des cardiomyopathies hypertrophiques. M ed Sci 1998 ; 14: 1054-66.

27. Satoh M, Takahashi M, Sakamoto $T$, $H$ iroe $M$, Marumo $F$, Kimura A. Structural analysis of the titin gene in hypertrophic cardiomyoathy: identification of a novel disease gene. Biochem Biophys Res Commun $1999 ; 262$ : 411-7.

28. Mair J. Cardiac troponin I and troponin $\mathrm{T}$ : are enzymes still relevant as cardiac markers? Clin Chim Acta 1997 ; 257: 99-115.

29. Rao KMK, Cohen, H.J. Actin cytoskeletal network in aging and cancer. M utation Research 1991 ; 256: 139-48.

30. Dustin ML, Cooper JA. The immunological synapse and the actin cytoskeleton: molecular hardware for T cell signaling. Nature $2000 ; 1: 23-32$

TIRÉS À PART

A. Fattoum.

\section{Summary}

Actin and actin-binding proteins II. The pathological aspects

The vast variety of cellular processes implicating the actin molecule, as either a component of the cytoskeleton or a major factor in muscle contraction and motility systems, requires the interactions of actin with its associated proteins. A fascinating example of actin-based motility in non-muscle cells is the case of the bacteria Listeria monocytogenes, which can invade eukaryotic cells and actively exploit actin from its host cell during infection and cell-to-cell spreading. Within the cell, the regulation of continuous cycle of assembly/ disassembly of actin is highly controlled by a large repertoire of proteins acting either individually or in concert, by sequestering monomers ( $\beta$-thymosin), capping (CapZ), cross-linking (filamin), bundling ( $\alpha$-actinin), severing (gelsolin) or stabilizing (tropomyosin) actin filaments. The development and function of multicellular organisms are dependent on the correct spatial and temporal organization of actin cytoskeleton, in response to appropriate environmental cues. Therefore, disruptions in the expression, the structure or the function of these proteins result in changes in cell behavior, leading to perturbation of actin architecture and in many cases, to the appearance of a wide range of pathogenic conditions and diseases. Indeed, disorganization of the actin cytoskeleton is part of the cell death program.

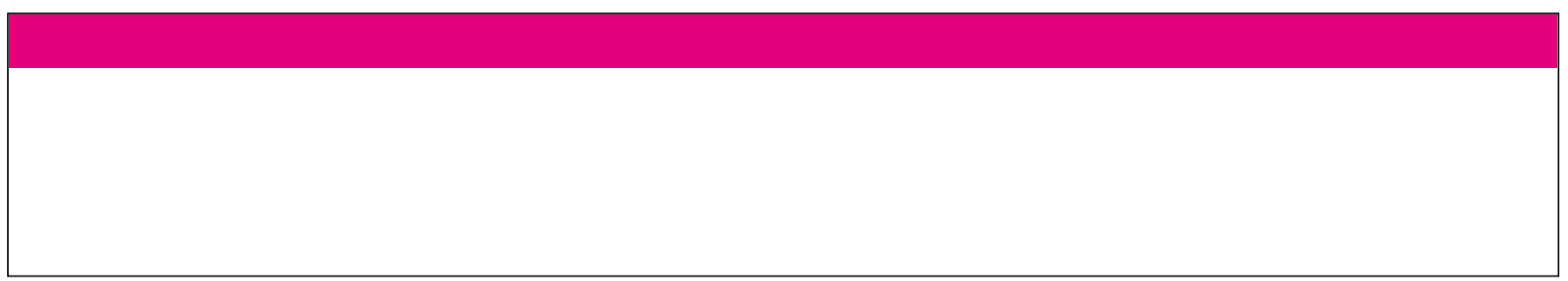

